Supporting Information :

\title{
Calcium Alginate Gel Beads Containing Gold Nanobipyramids for Surface-Enhanced Raman Scattering Detection in Aqueous Samples
}

Jiaming Chent,, Yuanyuan Yang ${ }^{\dagger,}$, Bingyong Lin*, Xueju Weit*, Zhenyu Lin ${ }^{* *}$, and Guolin Hong ${ }^{* *}$

$\dagger$ Department of Laboratory Medicine, The First Affiliated Hospital of Xiamen University, Xiamen Key Laboratory of Genetic Testing, Xiamen, Fujian 361005, China;

$\ddagger$ College of Chemistry, Fuzhou University, MOE Key Laboratory for Analytical Science of Food Safety and Biology, Fujian Provincial Key Laboratory of Analysis and Detection Technology for Food Safety, Fuzhou, Fujian, 350116, China

*E-mail: xuejuwei@126.com (Xueju Wei);

*E-mail: zylin@fzu.edu.cn (Zhenyu Lin);

*E-mail: 18860089899@139.com (Guolin Hong)

\section{Table of Content:}

Calculation of the analytical enhancement factor

Figure S1 SEM image of the Au NBPs/CAGB.

Figure S2 SEM images of Au NBPs/CAGB cross section with different concentrations of $\mathrm{Au}$ NBPs

Figure S3 Photographs of Au NBPs (10 nM)/CAGB exposed to environment.

Table S1 Table of experimental and reported Raman vibrational modes and their assignment of Rhodamine $6 \mathrm{G}$

Table S2 Table of comparison of the performance of the different SERS platforms (Rhodamine $6 \mathrm{G})$

Table S3 Table of experimental and reported Raman vibrational modes and their assignment of uric acid

Table S4 Table of comparison of the performance of the different SERS platforms (Uric acid) 


\section{Calculation of the analytical enhancement factor (AEF)}

The SERS analytical enhancement factor (AEF) was calculated according to the following equation: ${ }^{1-3}$

$$
\mathrm{AEF}=\left(\frac{\mathrm{I}_{\mathrm{SERS}}}{\mathrm{I}_{\mathrm{NRS}}}\right)\left(\frac{\mathrm{C}_{\mathrm{NRS}}}{\mathrm{C}_{\mathrm{SERS}}}\right)
$$

where $\mathrm{I}_{\mathrm{SERS}}$ and $\mathrm{I}_{\mathrm{NRS}}$ are the integrated intensities at $1363 \mathrm{~cm}^{-1}$ obtained by SERS and normal Raman scattering (NRS) spectrum. $\mathrm{I}_{\mathrm{SERS}}$ is 8204.7 , and $\mathrm{I}_{\mathrm{NRS}}$ is 301.9. $\mathrm{C}_{\mathrm{SERS}}$ and $\mathrm{C}_{\mathrm{NRS}}$ stand for the concentration of R6G solution used for SERS and normal Raman scattering measurements, $10^{-6} \mathrm{M}$ and $0.1 \mathrm{M}$, respectively. As a result, the calculated AEF of proposed Au NBPs/CAGB is about $2.7 \times 10^{6}$.
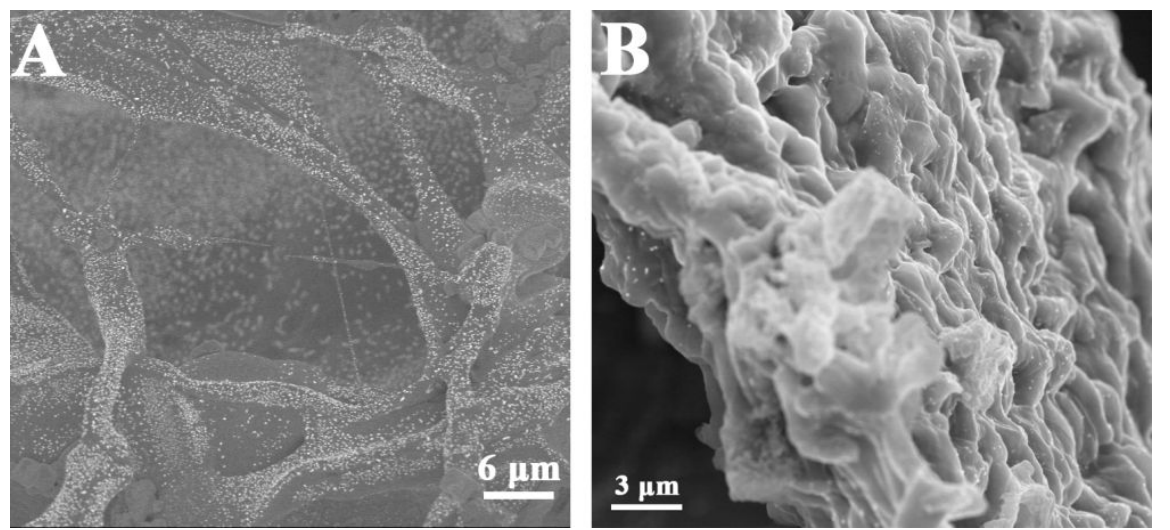

Figure. S1 (A) The SEM image of the Au NBPs/CAGB in large-scale. (B) The SEM image of the partial enlargement of a shrank and wrinkled cross section of Au NBPs/CAGB.

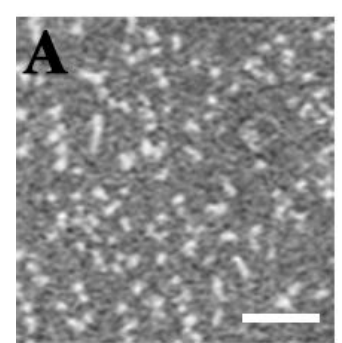

$5 \mathrm{nM}$

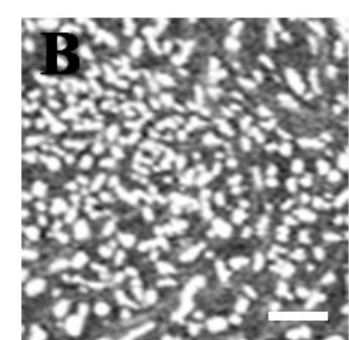

$10 \mathrm{nM}$

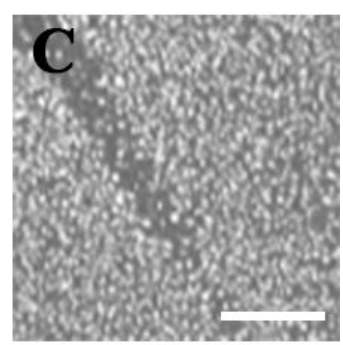

$15 \mathrm{nM}$

C $_{\text {Au NBP }}$

Figure. S2 SEM images of Au NBPs/CAGB cross section with different concentrations of $\mathrm{Au}$ NBPs: (A) $5 \mathrm{nM}$, (B) $10 \mathrm{nM}$, (C) $15 \mathrm{nM}$. . 


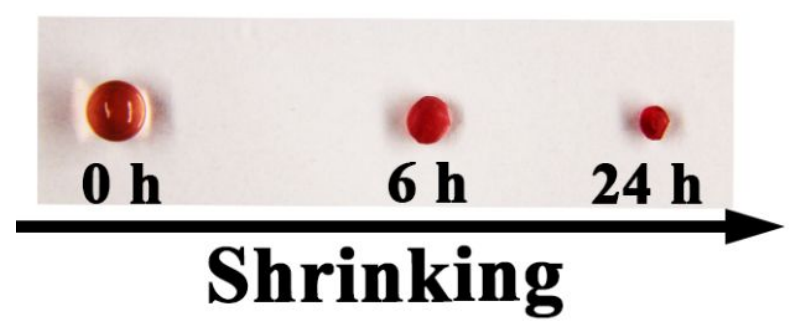

Figure. S3 The photographs of Au NBPs $(10 \mathrm{nM}) / \mathrm{CAGB}$ exposed to environment $\left(25^{\circ} \mathrm{C}\right.$, Relative humidity $60 \%$ ). The volume of bead decreased with the time increasing.

Table S1. Experimental and Reported Raman Vibrational Modes and Assignments of Rhodamine 6G $\left(\mathrm{cm}^{-1}\right)$

\begin{tabular}{ccc}
\hline \multirow{2}{*}{ Vibrational description } & \multicolumn{2}{c}{ Rhodamine 6G } \\
\cline { 2 - 3 } & Observed & Reported $^{1,4}$ \\
\hline C-C-C ring in plane bending & 613 & 613 \\
C-H out of plane bending & 777 & 775 \\
Aromatic C-C stretching & 1190 & 1183 \\
Aromatic C-C stretching & 1315 & 1312 \\
Aromatic C-C stretching & 1363 & 1364 \\
Aromatic C-C stretching & 1510 & 1512 \\
\hline
\end{tabular}

Table S2. Comparison of the Performance of the Different SERS Platforms (Rhodamine 6G)

\begin{tabular}{cccccc}
\hline SERS platform & $\begin{array}{c}\text { Detection } \\
\text { method and time }\end{array}$ & RSD & Detection Range & LOD & Ref. \\
\hline \multicolumn{1}{c}{ Rhodamine 6G } \\
$\begin{array}{c}\text { Graphene/Ag- } \\
\text { nanoflowers/PM } \\
\text { MA }\end{array}$ & $\begin{array}{c}\text { Immersed into } \\
\text { aqueous sample } \\
10 \text { minutes }\end{array}$ & N.A. & $10 \mathrm{fM} \sim 1.0 \mathrm{nM}$ & $10 \mathrm{fM}$ & 6 \\
\hline
\end{tabular}



Ag-nanosheet- grafted polyamide- nanofibers

Au nano-islands

Drop-casting, N.A.

$\mathrm{Cu}-\mathrm{Ag}$ layered periodic nanostructure

Roughened nano-Au film hours

microfluidic, N.A.

nanostructure

N.A. \& N.A.

N.A.

$1.0 \mathrm{nM} \sim 1.0 \mathrm{mM}$

$1.0 \mathrm{nM}$

$0.01 \mathrm{nM} \sim 10 \mathrm{nM}$

$0.01 \mathrm{nM}$

$8.8 \%$

$1.0 \mathrm{nM} \sim 10 \mu \mathrm{M}$

$1.0 \mathrm{nM}$

9

nano-Au film

$<11 \%$

$1.0 \mathrm{nM} \sim 10 \mu \mathrm{M}$

$0.07 \mathrm{nM}$

10

Verticallyaligned Au NRs assembled at liquid-liquid interface

Direct measure of liquid-liquid interface, N.A.

N.A.

$1.0 \mathrm{nM} \sim 10 \mu \mathrm{M}$

$1.0 \mathrm{nM}$

11
Superhydrophob ic Ag coated taro leaf
Drop-casting, 2 hours

$9.7 \%$

$10 \mathrm{nM} \sim 0.1 \mathrm{mM}$

$10 \mathrm{nM}$

12

\begin{tabular}{cccccc}
$\begin{array}{c}\text { Superhydrophob } \\
\text { ic surface }\end{array}$ & $\begin{array}{c}\text { Drop-casting, } \\
\sim \text { hour }\end{array}$ & N.A. & $75 \mathrm{fM} \sim 75 \mathrm{pM}$ & $10 \mathrm{aM}$ & 13 \\
$\mathrm{Au}$ & $\begin{array}{c}\text { Encapsulated the } \\
\text { sample for direct } \\
\text { measure, } 1 \text { minute }\end{array}$ & $6.57 \%$ & $1.0 \mathrm{nM} \sim 100 \mu \mathrm{M}$ & $0.4 \mathrm{nM}$ & $\begin{array}{r}\text { This } \\
\text { work }\end{array}$ \\
\hline
\end{tabular}


Table S3. Experimental and Reported Raman Vibrational Modes and Assignments of Uric Acid $\left(\mathrm{cm}^{-1}\right)$

\begin{tabular}{ccc}
\hline \multirow{2}{*}{ Vibrational description } & \multicolumn{2}{c}{ Uric acid } \\
\cline { 2 - 3 } & Observed & Reported $^{5}$ \\
\hline Ring in-plane deformation & 497 & 492 \\
CO bending/skeletal ring deformation & 641 & 634 \\
NH bending & 738 & 725 \\
CN bending/in-plane ring deformation & 809 & 811 \\
CN stretching & 1023 & 1016 \\
In-plane NH bending & 1208 & 1204 \\
CN stretching/ in-plane NH & 1354 & 1354 \\
C=O stretching & 1620 & 1653 \\
\hline
\end{tabular}

Table S4. Comparison of the Performance of the Different SERS Platforms (Uric acid)

Uric acid

\begin{tabular}{|c|c|c|c|c|c|}
\hline Ag colloid & N.A. \& N.A. & $8.5 \%$ & $10 \sim 1000 \mu \mathrm{M}$ & $1.0 \mu \mathrm{M}$ & 14 \\
\hline $\begin{array}{c}\text { Multilayered } \\
\mathrm{Au} / \mathrm{Ag} \text { Substrate }\end{array}$ & N.A. \& N.A. & N.A. & $100 \sim 1000 \mu \mathrm{M}$ & $100 \mu \mathrm{M}$ & 5 \\
\hline $\begin{array}{l}\text { Au nanoislands } \\
\text { on the cellulose } \\
\text { micro/nanofiber } \\
\text { s }\end{array}$ & $\begin{array}{l}\text { Dipped and dried, } \\
\text { N.A. }\end{array}$ & N.A. & $25 \sim 150 \mu \mathrm{M}$ ( in tear) & $25 \mu \mathrm{M}$ & 15 \\
\hline $\begin{array}{l}\text { Au nanoparticle- } \\
\text { coated paper }\end{array}$ & $\begin{array}{l}\text { Dipped and dried } \\
\text { prior, } 15 \text { minutes }\end{array}$ & $8.7 \% \sim 14.8 \%$ & $0 \sim 3500 \mu \mathrm{M}$ & $110 \mu \mathrm{M}$ & 16 \\
\hline $\begin{array}{c}\mathrm{Au} \\
\mathrm{NBPs} / \mathrm{CAGB}\end{array}$ & $\begin{array}{l}\text { Encapsulated the } \\
\text { sample for direct } \\
\text { measure, } 1 \text { minute }\end{array}$ & N.A. & $10 \sim 1000 \mu \mathrm{M}$ & $0.18 \mu \mathrm{M}$ & $\begin{array}{l}\text { This } \\
\text { work }\end{array}$ \\
\hline
\end{tabular}




\section{References}

(1) Canamares, M. V.; Chenal, C.; Birke, R. L.; Lombardi, J. R., DFT, SERS, and singlemolecule SERS of crystal violet. J. Phys. Chem. C 2008, 112, 20295-20300.

(2) Fu, Q.; Zhan, Z.; Dou, J.; Zheng, X.; Xu, R.; Wu, M.; Lei, Y., Highly reproducible and sensitive SERS substrates with Ag inter-nanoparticle gaps of $5 \mathrm{~nm}$ fabricated by ultrathin aluminum mask technique. ACS Appl. Mater. Inter. 2015, 7, 13322-13328.

(3) Lee, H. K.; Lee, Y. H.; Koh, C. S. L.; Phan-Quang, G. C.; Han, X.; Lay, C. L.; Sim, H. Y. F.; Kao, Y.-C.; An, Q.; Ling, X. Y., Designing surface-enhanced Raman scattering (SERS) platforms beyond hotspot engineering: emerging opportunities in analyte manipulations and hybrid materials. Chemical Society Reviews 2019, 48, 731-756.

(4) Kudelski, A., Raman studies of rhodamine $6 G$ and crystal violet sub-monolayers on electrochemically roughened silver substrates: Do dye molecules adsorb preferentially on highly SERS-active sites? Chemical Physics Letters 2005, 414, 271-275.

(5) Zhao, L.; Blackburn, J.; Brosseau, C. L., Quantitative detection of uric acid by electrochemical-surface enhanced Raman spectroscopy using a multilayered Au/Ag substrate. Analytical chemistry 2015, 87, 441-447.

(6) Qiu, H.; Wang, M.; Jiang, S.; Zhang, L.; Yang, Z.; Li, L.; Li, J.; Cao, M.; Huang, J., Reliable molecular trace-detection based on flexible SERS substrate of graphene/Agnanoflowers/PMMA. Sensor. Actuat. B-Chem. 2017, 249, 439-450.

(7) Qian, Y.; Meng, G.; Huang, Q.; Zhu, C.; Huang, Z.; Sun, K.; Chen, B., Flexible membranes of Ag-nanosheet-grafted polyamide-nanofibers as effective 3D SERS substrates. Nanoscale $2014,6,4781-4788$. 
(8) Fusco, Z.; Bo, R.; Wang, Y.; Motta, N.; Chen, H.; Tricoli, A., Self-assembly of Au nanoislands with tuneable organized disorder for highly sensitive SERS. J. Mater. Chem. C2019, 7, 6308-6316.

(9) Bai, S.; Serien, D.; Hu, A.; Sugioka, K., 3D microfluidic Surface - Enhanced Raman Spectroscopy (SERS) chips fabricated by all-femtosecond-laser-processing for real-time sensing of toxic substances. Advanced Functional Materials 2018, 28, 1706262.

(10) Wang, J.; Qiu, C.; Mu, X.; Pang, H.; Chen, X.; Liu, D., Ultrasensitive SERS detection of rhodamine $6 \mathrm{G}$ and p-nitrophenol based on electrochemically roughened nano-Au film. Talanta $2020,210,120631$.

(11) Kim, K.; Han, H. S.; Choi, I.; Lee, C.; Hong, S.; Suh, S.-H.; Lee, L. P.; Kang, T., Interfacial liquid-state surface-enhanced Raman spectroscopy. Nat. Commun. 2013, 4, 1-9.

(12) Huang, J.-A.; Zhang, Y.-L.; Zhao, Y.; Zhang, X.-L.; Sun, M.-L.; Zhang, W., Superhydrophobic SERS chip based on a Ag coated natural taro-leaf. Nanoscale 2016, 8, 11487-11493.

(13) De Angelis, F.; Gentile, F.; Mecarini, F.; Das, G.; Moretti, M.; Candeloro, P.; Coluccio, M.; Cojoc, G.; Accardo, A.; Liberale, C., Breaking the diffusion limit with super-hydrophobic delivery of molecules to plasmonic nanofocusing SERS structures. Nat. Photonics 2011, 5, 682-687. (14) Westley, C.; Xu, Y.; Thilaganathan, B.; Carnell, A. J.; Turner, N. J.; Goodacre, R., Absolute quantification of uric acid in human urine using surface enhanced Raman scattering with the standard addition method. Analytical chemistry 2017, 89, 2472-2477.

(15) Park, M.; Jung, H.; Jeong, Y.; Jeong, K.-H., Plasmonic schirmer strip for human tear-based gouty arthritis diagnosis using surface-enhanced Raman scattering. ACS nano $2017,11,438-$ 
443.

(16) Villa, J. E.; Poppi, R. J., A portable SERS method for the determination of uric acid using a paper-based substrate and multivariate curve resolution. Analyst 2016, 141, 1966-1972. 